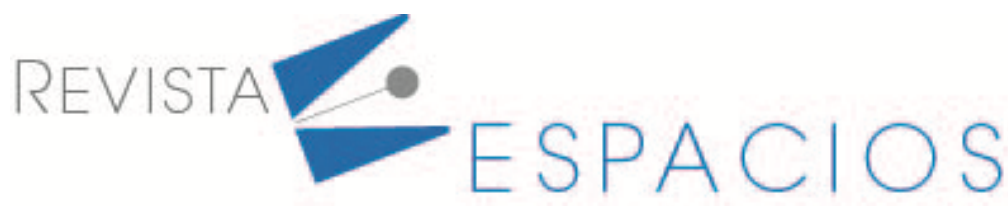

Vol. 42 (02) 2021• Art. 9

Recibido/Received: 26/10/2020 • Aprobado/Approved: 07/01/2021 • Publicado/Published: 31/01/2021

\title{
La localización y la situación financiera de las empresas hoteleras en las Ciudades Patrimonio de la Humanidad
}

\author{
The location and financial situation of hotel companies in World Heritage Cities
}

\author{
MUDARRA-FERNÁNDEZ, Ana B. ${ }^{1}$ \\ CAZALLO-ANTÚNEZ, Ana M. ${ }^{2}$
}

\begin{abstract}
Resumen
Esta investigación realiza una clasificación de la influencia de la situación financiera y las características organizacionales en la localización de los hoteles de las Ciudades Patrimonio de la Humanidad de Santiago de Compostela, Córdoba e Ibiza dada la importancia del turismo en el desarrollo económico y social. La metodología tiene enfoque cuantitativo, explicativa y utiliza la técnica del análisis discriminante, con variable categórica la localización y variables financieras y organizacionales resultando como variables explicativas del modelo el fondo de maniobra y el número de empresas del grupo.

Palabras clave: turismo cultural; administración de empresas; administración financiera; desarrollo económico y social
\end{abstract}

\begin{abstract}
The present research performs a classification of the influence of the financial situation and the organizational characteristics in the location of the hotels of the World Heritage Cities of Santiago de Compostela, Córdoba and Ibiza, given the importance of the tourism sector in econonomic and social development. The methodology used is framed within a quantitative, explanatory approach and uses the discriminant analysis technique, taking as a categorical variable the location and the financial and organizational variables, resulting as explanatory variables of the model the working capital and the number of companies in the group.

key words: cultural tourism; business management; financial administration; economic and social development
\end{abstract}

\section{Introducción}

El desarrollo local difiere del crecimiento económico puesto que el primero implica una mejora en las condiciones de vida de los ciudadanos siendo un catalizador de la sustentabilidad en el territorio (Carvalho y Moquete, 2011) completando lo anterior, autores como García, González y Acevedo (2019) consideran al turismo como motor de desarrollo económico de diferentes países al fomentar dinámicas económicas mediante la generación de divisas, empleo, creación de empresas, mejoras en los ingresos per cápita de las familias e incrementos en el PIB regional y nacional y desarrollo en la región. El hecho de que el desarrollo local se cimiente en el territorio y en sus gentes permite que mediante la realización de proyectos vinculados al desarrollo económico y social se conjugue el

\footnotetext{
${ }^{1}$ Profesor de la Universidad de Jaén. Departamento de Organización de empresas, Mareketing y Sociología. Universidad de Jaén. amudarra@ujaen.es

2 Profesor de la Universidad de Jén. Departamento de Organización de empresas, Mareketing y Sociología. Universidad de Jaén. acazallo@ujaen.es
} 
crecimiento económico local con el crecimiento del bienestar de la población en el largo plazo (Cantarero, Sanchís y Comeche, 2011). En esta línea, se precisa pues de una adaptación de los sectores económicos tanto a los territorios como a la sociedad para el diseño de la estrategia de desarrollo local correcta. Son muchos los territorios donde el turismo ha asumido un importante rol en materia de desarrollo local demostrando su capacidad de adaptación a los cambios sociales y del territorio, complementándose con el sector primario tras las crisis de este o incrementando la oferta de servicios turísticos basada en aspectos locales de naturaleza medioambiental, cultural o gastronómica convirtiéndose en una oportunidad estratégica para el desarrollo local (López y De Esteban, 2010).

De esta manera, el turismo es considerado como una alternativa para alcanzar el desarrollo, ya que ha permitido una diversificación económica generando oportunidades en los territorios, por lo que se ha convertido, a nivel local, en un elemento diferenciador que le proporciona al destino la capacidad de competir con otros lugares (Wallingre, 2007). En esta línea, Narváez (2014) considera al turismo como una oportunidad estratégica que va más allá del turismo de masas y destaca la existencia de otras áreas potenciales como pueden ser el turismo natural, patrimonial o cultural que además produce un efecto de arrastre sobre otros sectores económicos, como, por ejemplo, el comercio.

Es tal la importancia estratégica que presenta el turismo, tanto en destinos de masas como en aquellos destinos con riqueza singular, que en 1993 un grupo de ciudades que habían sido declaradas por la UNESCO como Patrimonio de la Humanidad crean el grupo de Ciudades Patrimonio de la Humanidad, distinción que tiene como objetivo la perservación y salvaguarda del patrimonio territorial para que este pueda ser disfrtutado por las generaciones venideras tal y como se ha heredado de nuestros ancestros. En otras palabras, la inclusión o categorización de una ciudad como World Heritage Site (WHS) permite la preservación del patrimonio y el reconocimiento de un alto nivel de calidad patrimonial, lo que se traduce en un atractivo turístico para dichas ciudades (Pérez, Medina-Viruel, López-Guzmán y Muñoz-Fernández, 2020), contribuyendo al desarrollo económico de la localidad mediante el desarrollo de un sector turístico en la zona, lo cual se traduce en la creación y crecimiento de empresas dedicadas a servicios turísticos.

Junto a lo anterior es preciso mencionar que el patrimonio cultural permite mostrar la identidad de un territorio generando un elemento diferenciador atractivo mediante el desarrollo de la actividad cultural sustentada en el patrimonio que convierte a este en un preciado bien turístico (González y López-Guzmán, 2016). De hecho, autores como Cárdenas, Pulido y Mudarra (2015) destacan que la declaración del destino como Ciudad Patrimonio de la Humanidad beneficia a la economía local debido al incremento del gasto turístico en el territorio.

Desde una perspectiva nacional, España cuenta con un total de 15 ciudades Patrimonio de la Humanidad que se pueden clasificar en tres grupos (planetas, cometas y satélites) atendiendo al reto u objetivo marcado a futuro por estas ciudades (Braintrust, 2019). De esta manera, el grupo planetas está compuesto por Córdoba, Santiago de Compostela e Ibiza, objeto del presente estudio; tiene asumido como reto crecer sin saturarse ya que se trata de ciudades caracterizadas por alojar a un gran número de viajeros (superior a 600 mil turistas anuales) en su mayoría extranjeros y que cuentan con facilidades acceso lo cual genera un volumen alto de ocupación procedente del sector. Otra característica importante de estas ciudades, motivo por el cual atrae a los turistas, es el patrimonio en sí, lo que se traduce en que exista la necesidad de aumentar la recurrencia del destino y gestionar el incremento de la presión turística sin que llegue a saturarse. Por otro lado, las ciudades satélites (Tarragona, Alcalá de Henares, Segovia, Ávila, Toledo, Salamanca y La Laguna) tienen como reto la capacidad de convertirse en destino final, por lo que su objetivo a cumplir es aumentar la estancia e incrementar el número de turistas extranjeros. Estas ciudades, dentro del cómputo global de Ciudades Patrimonio de la Humanidad, 7 de 15 , se caracterizan por tener un volumen medio de viajeros entre 200 y 600 mil turistas que generalmente se 
suelen alojar en municipios grandes y accesibles desde otros núcleos turísticos importantes. Sin embargo, la estancia media en estos destinos es corta, con escasez de turistas extranjeros y una oferta menor de alojamientos hoteleros que en el resto de grupos. El tercer grupo los componen Cáceres, Mérida, Úbeda, Cuenca y Baeza, también denominadas cometas, presentan como principal reto el incremento de su relevancia turística, para lo que es necesario equilibrar la oferta con la demanda mediante la atracción de un mayor público, principalmente extranjero y, además, incrementar la estancia media de sus visitantes. Estas ciudades se caracterizan por tener un bajo número de turistas, especialmente extranjeros, que se suelen alojar en grandes destinos turísticos con los que no se tiene una buena accesibilidad y se tiene un bajo grado de ocupación (inferior a 55\%). Es por esto que para ganar relevancia turística es importante la realización de medidas de accesibilidad con otros puntos turísticos que ostenten una mayor atracción turística y la creación de actividades turísticas actractivas que incrementen el grado de ocupación y la estancia media.

A continuación, se describe brevemente la relevancia patrimonial de las ciudades de Santiago de Compostela, Córdoba e Ibiza. En el caso de Córdoba, fue declarada en 1994 como Ciudad Patrimonio de la Humanidad, siendo la ciudad con más monumentos declarados Patrimonio de la Humanidad de España, lo que la caracteriza como destino atractivo cultural turístico (Morales-Fernández y Lanquar, 2014), y el cual se compone por 4 monumentos, tangibles e intangibles, galardonados por la UNESCO como Patrimonio de la Humanidad como son su Mézquita-Catedral (1984), centro histórico (1994), Fiesta de los Patios (2012) y Medina-Azahara (2018). Este hecho atrae la atención de un gran número de turistas donde en el $45 \%$ de los casos según Moral, López, Menor y Rueda (2020) se caracterizaban por ser mujeres con una edad comprendida 30-49 años, de nacionalidad española, con formación universitaria, asalariada a tiempo completo y con unos ingresos de entre 700 y 1.500 euros, que eligen acudir al destino por cuatro razones motivacionales (cultural, social, patrimonial y ocio). Por otro lado, Morales-Fernández y Lanquar (2014) determinan que en 2010 el perfil del turista que acudía a Córdoba era principalmente europeos de género masculino, con edades comprendidas entre 30-44 años, con estudios superiores, con unos ingresos medios que oscilan entre 1001-1500€ y que los motivos que provocan su viaje son vacaciones y excursiones.

Con respecto a la ciudad de Santiago de Compostela, la capital de la Comunidad Autónoma de Galicia, cabe destacar que es el destino final del Camino de Santiago, de suma importancia no solo de caracter patrimonial sino también de carácter religioso, lo que implica que a este destino lleguen miles de turistas cada año, cifras que se ven incrementadas en año Xacobeo. Santiago de Compostela fue galardonada como Ciudad Patrimonio de la Humanidad desde 1985 cuando fue declarado su casco histórico como Patrimonio de la Humanidad por la UNESCO dada su gran belleza y valor patrimonial (Martínez, 2016). Para el caso de Santiago el valor monumental, así como la integridad y su fuerte carácter espiritual le hace ser uno de los centros más importantes de la cristiandad, tanto por tamaño como por ser el destino principal del movimiento religioso y cultural de Europa, lo que la convierte en un fuerte motor de atracción de turistas. Todo esto ha provocado un incremento del empleo, un aumento de los productos turísticos culturales y la atracción de un turismo fiel. Además, el mismo autor destaca la repercusión positiva sobre la economía gallega que tiene el turismo cultural-religioso en Santiago gracias a las buenas comunicaciones existentes con el resto de Galicia, permitiendo el desarrollo del sector en el conjunto de la comunidad autónoma. Finalmente, y con lo que respecta a esta ciudad se ha de indicar su marcado carácter como líder en el fomento de la sostenibilidad de su localización, al realizar fuertes programas de mantenimiento de su patrimonio para que las generaciones venideras puedan acceder a su numeroso valor patrimonial como nosotros la heredamos de generaciones pasadas.

Por otro lado, Ibiza tiene un territorio de $542 \mathrm{~km}^{2}$, es uno de los destinos turísticos más destacados a nivel nacional e internacional desde los años 30, sin embargo, no es hasta 1999 cuando es inscrita en la lista de Patrimonio Mundial bajo la denominación de "Ibiza, Biodiversidad y Cultura" por la UNESCO convirtiéndose así en Ciudad Patrimonio de la Humanidad. Según Azpelicueta, Ramón y Abril (2018), dicha catalogación se debe a 
sus numerosos monumentos como son Dalt Vila, le necrópolis fenicio-púnica de Puig des Molins, el asentamiento sa Caleta y las praderas de posidonia oceánica situada dentro de la Reserva Natural de Ses Salines ubicada entre Ibiza y Formentera, las cuales ayudaron al impulso de la visibilidad de su patrimonio, al mismo tiempo que a la diversificación del sector con respecto al turismo de sol y playa predominante en la isla durante el periodo estival.

Pese a la importancia patrimonial, el caso de Ibiza es diferente, ya que según afirman Gurrea y Herrera (2009), como se cita en Azpelicueta, Ramón y Abril (2018), el turismo cultural tiene escasa relevancia en la elección de los turistas en el momento de elección de Ibiza como destino de vacaciones, tal y como muestran las escasas cifras de visitantes de museos y centros de interpretación, siendo el turismo de sol y playa el que atrae a un mayor número de turistas al destino. A pesar de ello, se han desarrollado diversas acciones para impulsar el atractivo de la zona monumental, aunque su población sigue siendo escasa y envejecida al no ser atractiva la ubicación para nuevos residentes en pro de otras zonas de la isla en donde se desarrolla un turismo más enfocado al turista de lujo.

En definitiva, la presente investigación explica y predice la clasificación de las empresas hoteleras de las WHS españolas categorizadas como ciudades planetas (Santiago de Compostela, Córdoba e Ibiza) a partir de los datos presentados para el año 2018. Dicha clasificación se realiza tomando como variable categórica o clasificatoria a la localización, distinguiendo 3 grupos: las empresas localizadas en Santiago de Compostela, las empresas localizadas en Córdoba y las empresas localizadas en Ibiza. Por otro lado, para el desarrollo del modelo se toman como variables explicativas o discriminatorias variables organizacionales y financieras.

\section{Marco teórico}

La localización empresarial es considerada como uno de los principales factores que determinan la rentabilidad de las empresas al influir de manera directa en su capacidad de generar valor para el cliente final, por lo que su determinación se considera una decisión de carácter estratégico al depender de esta el éxito de la empresa y estar directamente relacionada con otras variables como son la ocupación, la intensidad competitiva y la estacionalidad del destino turístico donde la empresa se desea ubicar (Graça, Couto, Botelho y Faias 2014 y Gémar 2014, como se cita en Lado, Otero y Vivel, 2014, Jenny y Weber, 1976 y Neumann, Böbel y Heid, 1982). Cabe destacar que la localización cobra especial importancia dentro del sector turístico, ya que es considerada como un elemento clave para la generación de valor añadido respecto al servicio prestado o suministrado.

Más concretamente, en el sector turístico cobra especial interés para la generación de valor añadido del servicio las variables espacio y tiempo, es decir, la capacidad de ofrecer un servicio en un lugar determinado (en este caso, en las Ciudades Patrimonio de la Humanidad) y en un momento concreto que generalmente suele coincidir con el viaje del turista. Estas dos variables, espacio y tiempo, pertenecientes a la cadena de suministro de cualquier empresa son importantes en el sector turístico al recoger la necesidad de satisfacer los requerimientos de los viajeros que acuden a un destino turístico en un momento concreto. Es por esto, que los stakeholders y, entre ellos, los gestores de destinos, tienen la necesidad de ofrecer una gama amplia de servicios de ocio y entretenimiento a lo largo de todo el año, con el fin de llegar no solo a un mayor número de turistas, sino también de persuadirlos para que incrementen su estancia en la localidad.

Completando lo anterior, Lado et al. (2014) afirman que la localización es una variable de especial interés al ser tomada mediante decisiones estratégicas no modificables a corto plazo que determinan la rentabilidad del establecimiento hotelero y, por ende, se considera factor clave en el éxito empresarial de los establecimientos hoteleros. Es tal la importancia estratégica de la variable que influye de forma directa no solo en el grado de ocupación y en la estancia media que tendrán los visitantes al destino, sino que también determina las finanzas de los establecimientos hoteleros a través de su influencia en el coste de los recursos necesarios para la 
producción del servicio, como es el caso del valor del suelo donde se han urbanizado las infraestructuras de alojamiento, el coste de las infraestructuras hoteleras, el coste de los suministros, coste de personal, etc.

También cabe mencionar que la localización está directamente relacionada con la capacidad competitiva concentrada en el destino, ya que a mayor nivel de concentración empresarial menor competencia existe en el destino. Es por esto que, como expone Porter (2008), si un hotel se localiza en un entorno de baja concentración del mercado, esto puede repercutir de forma negativa en su rentabilidad al caracterizarse el sector hotelero por presentar altos niveles de competencia. Sin embargo, otros autores como Bain (1951) visto en Raguseo, Vitari y Pig (2020) y Weis (1974) como se cita en Choi y Lee (2020) determinan que son otras variables como la estructura empresarial y el comportamiento las que influyen en la rentabilidad y concentración del mercado.

Sin embargo, la escasez de concentración empresarial puede presentar relaciones positivas, si ocurre en destinos donde existen altos índices de ocupación, reducción de la estacionalidad o competidores con poco poder de influencia sobre los precios. Además, la baja concentración supone un incremento en la competitividad de las empresas del destino, las cuales tendrán que recurrir a estrategias de diferenciación y adaptabilidad a las necesidades del turista con el objetivo de proporcionar a este el mayor valor añadido posible, favoreciendo de esta forma al desempeño realizado por la empresa. Pero la importancia de la localización en el sector hotelero no solo recae en la influencia sobre los resultados empresariales, sino que la toma acertada de esta decisión estratégica proporcionará a la empresa de recursos atractivos para el turista, lo que afecta a la creación de valor añadido mediante el aprovechamiento tanto de las capacidades de la empresa como de los recursos ubicados en el entorno próximo.

Además, una empresa hotelera localizada en un entorno con baja concentración de mercado puede generar un incremento en el nivel de competencia existente al haber un mayor número de empresas que luchan por atraer la atención del cliente, pero al mismo tiempo, permite acceder a mejores recursos, a un mayor nivel de demanda y a externalidades derivadas de la actuación de los competidores mediante la transferencia de know-how, concentración de mercado laboral especializado en el sector, colaboración en el uso de infraestructuras, contactos o aspectos de índole institucional que generan externalidades que se potencian en zonas denominadas clúster como pueden ser las Ciudades Patrimonio de la Humanidad de Santiago de Compostela, Ibiza y Córdoba, sin embargo, no todas las empresas de estas ciudades se van a aprovechar de estas externalidades ya que aquellas que no formen parte del clúster no podrán contar con estas ventajas.

En lo que respecta al sector hotelero en España, la afiliación a una cadena hotelera o el establecer acuerdos de colaboración con cadenas de hoteles independientes es un hecho habitual enfocado a la reducción de las limitaciones existentes en la mejora de la eficiencia de los activos. Este hecho se agrava en el caso de las micropymes, las cuales presentan reducidos niveles de rentabilidad, como consecuencia de su reducido tamaño, y la dificultad de acceder a economías de escala. Ya que para que estas empresas puedan realizar de forma normal su actividad empresarial van a requerir inventir un mayor volumen de recursos en activos fijos, lo que genera que sus activos circulantes sean reducidos, con lo que estarían generando un menor volumen de liquidez $y$, por ende, un aprovechamiento del mismo inferior que el que realizan los hoteles de gran tamaño. Esto se debe a que a mayor nivel de liquidez menor será la necesidad de endeudarse para poder hacer frente a sus deudas a corto plazo, por lo que se puede deducir que a mayor tamaño de la empresa menor será la necesidad de endeudamiento. Por tanto, si los hoteles con microtamaño tienen menos disponibilidad de liquidez, eso generará que estos se tengan que endeudarse para poder hacer frente a sus deudas, al mismo tiempo que presentan una mayor relación entre fondos propios y deuda a corto plazo. Pero si se tiene en cuenta que el ratio de fondos propios junto con el de deuda a corto plazo es alto, esto señala que las micropymes tienden más a endeudarse a largo plazo que a corto plazo, lo que se puede justificar por la dificultad o reticencia existente a acudir a 
financiación procedente de inversores externos unido, a la dificultad de preveer la devolución de deuda a corto plazo.

Por otro lado, y siguiendo a Secall (2010), quien explica que los clústeres y su capacidad de generar ventajas competitivas como consecuencia de la aglomeración de empresas dedicadas a un mismo sector y en un punto geográfico específico, permite la generación de decisiones estratégicas. En cuanto al sector turístico, se debe afirmar que el hecho de tener un gran número de empresas dedicadas al ámbito patrimonial y cultural dentro de un destino geográfico concreto o específico, como es el caso de las Ciudades Patrimonio de la Humanidad (Santiago de Compostela, Córdoba e Ibiza), permite la creación de clústeres empresariales que mejoran la competitividad empresarial y permite generar ventajas competitivas de manera sostenible para el conjunto de las empresas debido al aprovechamiento de sinergias, al mismo tiempo que se comparten conocimientos y se abordan problemas parecidos. Asi mismo, a partir de los clústeres surgen múltiples relaciones de interdependencia que fomentan la competitividad de la empresa y del clúster en su conjunto mediante la transferencia de conocimiento, personal, recursos, entre otros aspectos. Sin embargo, para la creación de un clúster es necesario que la empresa se ubique dentro de un entorno dinámico e innovador que incentive el emprendimiento. De esta manera, la localización se convierte en una variable prioritaria en la creación de clústeres empresariales.

Por otro lado, en relación a las variables discriminatorias o clasificatorias, número de empresas incorporadas al grupo y ratio de fondo de maniobra, cabe mencionar que para el caso del sector turístico la existencia de grupos empresariales es un hecho habitual. Esto se debe a que muchas empresas pertenecen a grandes cadenas hoteleras, lo que permite aprovechar economías de escala, de alcance y sinergias sobre todo en zonas como Ibiza y Santiago de Compostela, donde se conjuga el turismo cultural-patrimonial con el turismo de ocio nocturno o religioso, respectivamente. Sin embargo, para el caso de Córdoba es más frecuente la existencia de pequeñas y medianas empresas, en lugar de grandes cadenas hoteleras, en lugares cercanos a sus monumentos.

Dicho carácter dinámico e innovador que permite el fomento del emprendimiento en un destino, no solo debe crear nuevas empresas sino que debe ayudar en la consolidación y crecimiento de las empresas hoteleras ya existentes. Sin embargo, a las empresas ubicadas en un entorno competitivo no solo le influye en su crecimiento su entorno externo sino que también tienen una gran repercusión en ellas sus propias finanzas. Esto se debe a que las empresas deben de poseer capacidad financiera positiva para poder hacer frente a las inversiones necesarias para realizar un incremento de su campo de actividad o procurar su consolidación en el sector. Para determinar la capacidad financiera de la empresa a corto plazo es necesario determinar el fondo de maniobra de la misma, ya que permite determinar cual es la capacidad de la empresa para hacer frente a sus compromisos a corto plazo, es decir, qué capacidad tiene la empresa para hacer frente a los pagos generados de su ciclo de explotación.

Para poder determinar la capacidad financiera de la empresa hotelera, con el fin de hacer frente a sus pagos más inmediatos, se determina el fondo de maniobra mediante la diferencia entre el activo y el pasivo circulante (Fondevila, 1986 como se cita en Hontalvilla, Lado y Vivel, 2018). Es decir, se identifican cuales son los bienes y derechos líquidos o que pueden convertirse en dinero en un espacio temporal inferior a un año y que se compone principalmente por el dinero que se le adeuda a la empresa por parte de clientes y deudores, las existencias que posee la misma y el dinero líquido que posee en caja o bancos. Pero a estos activos se le deben de sustrater, para determinar el fondo de maniobra, los pasivos circulantes, es decir, las deudas que posee la empresa y que deben de ser pagadas en un plazo inferior a un año entre las que se deben de destacar los créditos comerciales concedidos por proveedores y acreedores, los créditos bancarios conformados por préstamos, líneas de crédito y descuentos de efectos, los pagarés de empresas y el factoring. Dicha resta debe de ser positiva en la mayoría de los casos si la empresa se encuentra en situación de equilibrio financiero. 
Sin embargo, y según lo expuesto por Hontalvilla, Lado y Vivel (2018), para el sector hotelero el fondo de maniobra presenta una serie de diferencias con el resto de sectores económicos, ya que para las finanzas de este tipo de empresas el hecho de tener un fondo de maniobra negativo y sin embargo mantener el equilibrio financiero de la empresa es una característica habitual. Esto se debe principalmente a dos razones, la primera consistente en el tiempo o duración del ciclo de explotación de la actividad hotelera. Generalmente, el periodo medio de pago a proveedores hoteleros es superior al ciclo de explotación, ya que este tipo de empresas reciben sus ingresos e incrementos de caja de forma inmediata una vez prestado el servicio al tener una mayoría de clientes que suelen pagar al contado. Este hecho permite a la empresa disponer de altos volúmenes de liquidez mientras que hacen frente a sus pagos que presentarán plazos de pago de 30,60 o 90 días generalmente.

Por otro lado, se encuentra la segunda razón por la cual es habitual que las empresas hoteleras posean ciclos de explotación negativos y que se justifica en la composición del activo circulante. Puesto que en las empresas hoteleras el nivel de liquidez es superior que las deudas a las que tiene que hacer frente a corto plazo, esto indica que el riesgo de no poder hacer frente a las mismas es inferior y, por tanto, la calidad del activo corriente es más elevada para el caso de los hoteles que para cualquier otro sector económico. Esto hecho, tranquiliza a los proveedores de esta industria e inclusive anima a otros proveedores potenciales a trabajar con empresas hoteleras. Se ha de indicar que esto ocurre en situaciones económicas normales, sin embargo, en la actualidad las empresas hoteleras están sufriendo una grave crisis como consecuencia de la pandemia mundial de la Covid19 y, aunque se haya indicado que el riesgo de que los proveedores no cobren es inferior al resto de industrias, en esta situación se debe de indicar la existencia de riesgo al no disponer las empresas hoteleras de liquidez suficiente como consecuencia de la drástica reducción de la demanda de sus servicios.

Para el caso concreto de esta investigación el ratio de fondo de maniobra, de acuerdo con lo expuesto por la base de datos SABI (Sistemá de Análisis de de Balances Ibérico), ha sido calculado mediante el cociente entre la sustracción entre el activo corriente y pasivo corriente y el total del activo (SABI, 2020). Es decir, el ratio de fondo de maniobra se constituye por la relación existente entre la deuda o adeudo con que se queda la empresa después de hacer frente a sus deudas más inmediatas con los ingresos y recursos disponibles de la empresa a corto plazo y el total de recursos, bienes y derechos que posee la empresa en su totalidad.

En lo que respecta a las variables relacionadas con la situación financiera, cabe mencionar que tras la crisis económica y financiera de 2008, se realizaron un gran número de estudios científicos que buscan predecir el fracaso empresarial mediante la elaboración de modelos basándose en la información contable y financiera de las empresas (García-Gallego y Mures-Quintana, 2013) y concretamente, existe gran variedad de estudios que han utilizado las razones financieras para el análisis financiero. Se destacan trabajos como el realizado por López (2006), donde se analiza la probabilidad de quiebra en las empresas a partir de los balances y las razones financieras utilizando la técnica del análisis discriminante multivariado (ADM) para así identificar y evaluar los factores de riesgo y fracaso empresarial. Otro ejemplo, es el estudio realizado por Rico (2015) que analiza, mediante un modelo de elección binaria, las diferencias respecto al perfil económico-financiero de las empresas que a pesar de la crisis han seguido siendo rentables frente a las que no. Sin embargo, existen otras variables que se ven afectadas por la situación financiera y otros aspectos propios de la organización que pueden derivar en éxito o fracaso como es el caso de la localización.

El análisis financiero empresarial pertenece al área de la gestión financiera y se encarga de estudiar las conexiones entre la realidad de los hechos y los fenómenos monetarios que ocurren dentro de las empresas, así como de sus resultados (Gil, 2004). En relación al desarrollo de análisis financiero hay que mencionar la existencia de dos corrientes claramente diferenciadas. De un lado, se encuentran los que se basan en la información presentada en los estados financieros para comprender la situación financiera de las empresas utilizando la información contable y, de otro lado, los que enfatizan en cuestiones más holísticas haciendo hincapié en 
aspectos cualitativos (internos y externos) que influyen en las operaciones empresariales (Puerta, Vergara y Huertas, 2018).

Además, dentro de los que se basan en la información contable, existen 3 métodos para realizar el análisis financiero: el método de los porcentajes, el método de las diferencias y el método de ratios que es el que se va a utilizar en este trabajo. Dentro del método de porcentajes hay que distinguir entre los porcentajes horizontales y los verticales. El primero de ellos, analiza la variación de las diferentes partidas del balance y la cuenta de resultados en relación con el periodo anterior permitiendo conocer su evolución en el tiempo, mientras que, el segundo informa del peso de cada partida respecto al total permitiendo comparaciones con otras empresas. Respecto al método de las diferencias, hay que mencionar que se trata de un método estático basado en la agrupación de las masas patrimoniales del balance de acuerdo a un criterio funcional, con el fin de poder comparar las masas de activo y pasivo. La diferencia entre activo y pasivo es una medida del equilibrio financiero de forma que si es negativo se considera que la empresa se encuentra en una situación de desequilibrio y viceversa (Corona, Bejarano y González, 2017).

En relación a los ratios financieros, que es donde se enmarca este trabajo, hay que resaltar que desde una perspectiva conceptual, un ratio es una razón, es decir, un cociente entre diferentes partidas del balance y la cuenta de pérdidas y ganancias, que genera información relativa a la situación financiera de la empresa y facilita la toma de decisiones a los diferentes agentes económicos que se encuentran dentro y fuera de ella (Aching, 2006). De esta manera, mediante las ratios se puede determinar el desempeño financiero de las empresas, establecer acciones correctivas y preventivas relacionadas con la liquidez, la solvencia y la rentabilidad (MartínezCaraballo, Cazallo-Antúnez, Meñaca-Guerrero y Uribe-Urán, 2020). Gracias a esta información, el gestor puede, mediante el análisis de las ratios, conocer la posición competitiva de la empresa, los puntos fuertes o débiles de la misma o identificar las desviaciones entre lo presupuestado y la realidad. Asimismo, el accionista puede determinar la viabilidad del negocio o, el proveedor puede conocer la capacidad de la empresa para hacer frente a sus obligaciones (Gómez-Bezares, Madariaga y Santibáñez, 2004).

Por otro lado, desde una perspectiva práctica, el análisis mediante ratios permite realizar análisis de series temporales donde se estudia el comportamiento de las ratios de una empresa durante un periodo largo, de manera que permita calcular una tendencia que pueda servir de base para la realización de predicciones. Además, se pueden realizar análisis interempresas o análisis cruzado donde comparar las ratios de una empresa con otras similares o con la media del sector. Y, por último, se pueden realizar combinaciones entre los dos tipos de análisis anteriores (Ortega, 2010).

Dentro de las ratios financieras hay que distinguir dos grupos atendiendo al plazo. De esta forma, aquellas ratios con una perspectiva cortoplacista, cuyo objeto es el análisis de la convertibilidad de los activos en liquidez, están orientados a medir la capacidad de una empresa para hacer frente a los compromisos con un plazo inferior a un año o al periodo medio de maduración. En este grupo se sitúan las ratios de liquidez, las ratios de cobrabilidad y las ratios de cobertura de los gastos financieros y del principal de la deuda. Por el contrario, los que poseen una perspectiva de largo plazo se basan en el análisis de la estructura financiera y la correlación con la estructura económica de la empresa, entre los cuales se encuentran las ratios de independencia y garantía financiera, las ratios de endeudamiento, las ratios de capitalización y las ratios que permiten el seguimiento de la financiación (Rivero y otros, 1998 como se cita en Nevado y López, 2009).

Sin embargo, para la realización de esta investigación se han empleado las ratios financieras según la categorización Informa de Sabi junto con variables organizacionales las cuales se presentan en la tabla 1. 
Tabla 1

Ratios financieros según Informa y variables de empresa
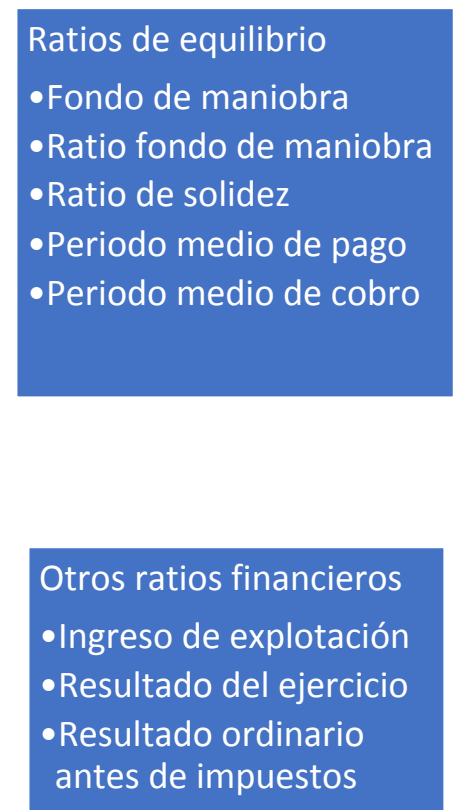

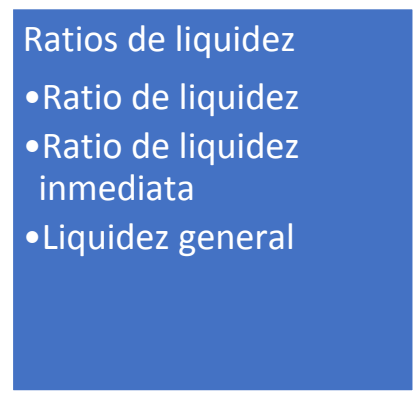

Ratios de resultados

-Rentabilidad económica (ROA)

-Rentabilidad de explotación

- Rentabilidad financiera (ROE)

- Rentabilidad económica

-Rentabilidad financiera
Ratios de endeudamiento

-Ratio de porcentaje de endeudamiento

- Ratio de coste medio de la financiación externa - Ratio de cobertura del servicio de la deuda -Endeudamiento

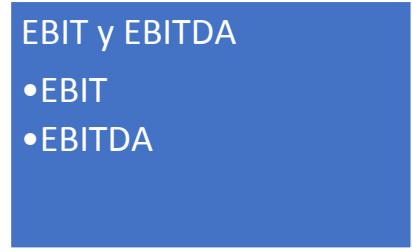

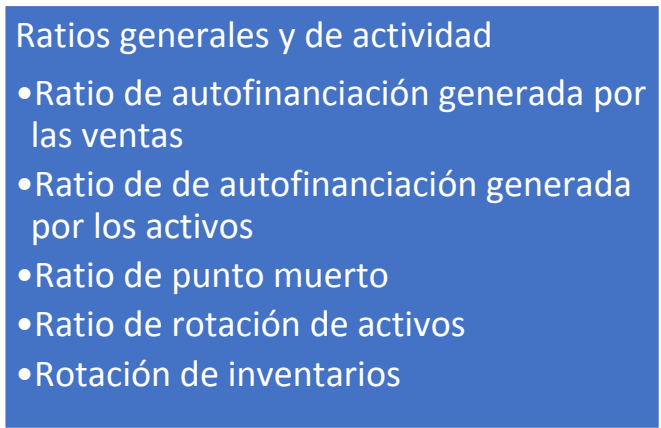

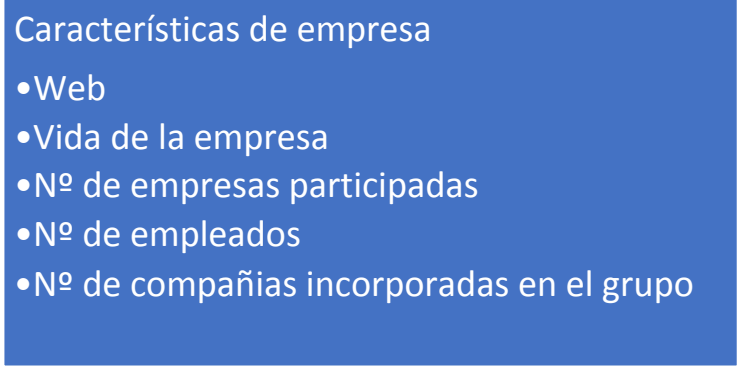

Fuente: elaboración propia a partir de Sabi 2020

\section{Metodología}

La investigación de este estudio se enmarca dentro del paradigma positivista puesto que trata de analizar o explicar la evolución financiera de las empresas dentro de un marco cuantitativo, empírico-analítico, racionalista y dentro del ámbito empresarial o gerencial (Ricoy, 2006). Concretamente, mediante el análisis de la evolución de la situación financiera se puede establecer predicciones mediante el uso de las ratios financieras favoreciendo el diseño de estrategias empresariales. La investigación se enmarca dentro del enfoque cuantitativo puesto que se basa en la recolección de datos numéricos para la probación de hipótesis y el análisis estadístico, para así establecer normas de comportamiento y poder probar teorías (Hernández, Fernández y Baptista, 2014). En este caso, se utilizará como variables explicativas las ratios financieras de Informa y características organizacionales.

La investigación es de tipo analítica-explicativa puesto que determina las pautas internas de un fenómeno y permite acceder a un conocimiento más amplio y extenso del fenómeno estudiado (Hurtado, 2010). El diseño de la investigación es no experimental ya que no se realiza manipulación de los datos por parte de los autores, tomándose estos directamente de la base de datos (Hernández et al., 2014). De esta manera, se utilizan un total 
de 107 observaciones repartidas en tres grupos atendiendo a la localización, es decir, al lugar donde esté situada la empresa (Córdoba, Santiago de Compostela e Ibiza).

Para la determinación de las observaciones pertenecientes a la muestra se utiliza la técnica de muestreo no probabilístico de tipo accidental o consecutivo, ya que dentro de la población hay observaciones que no muestran la totalidad o parcialidad de los datos de las variables (ratios financieros) siendo elegidas por los autores aquellas empresas que sí presentan totalidad de los datos (Otzen y Manterola, 2017). Debido a esto, el tamaño muestral resultante se desglosa en 50 empresas en Ibiza, 24 en Córdoba y 33 en Santiago de Compostela, las cuales fueron obtenidas de la base de datos SABI dentro del grupo IAE, concretamente, en el 6810 denominado Servicio de hospedaje en hoteles y moteles.

Respecto a la técnica estadística utilizada, en la presente investigación se realiza un análisis discriminante multivariado mediante la inclusión por pasos, utilizando como variable categórica la localización, es decir, la Ciudad Patrimonio de la Humanidad donde se encuentran las empresas y como variables discriminatorias las razones financieras de acuerdo a la nomenclatura Informa, junto a otras características organizacionales.

\section{Resultados}

A continuación, se presentan los resultados del análisis discriminante tomándose como variable categórica la localización y variables discriminantes las razones financieras y otras variables de empresa.

En primer lugar, se realiza con carácter previo al desarrollo del análisis discriminante se lleva a cabo la prueba $\mathrm{M}$ de Box (tabla 1) donde se determina la igualdad de matrices de covarianza rechazándose la hipótesis nula por la que se establece que las matrices de covarianza poblaciones son iguales al presentar una significancia de 0.000 .

Tabla 1

Prueba M de Box

\begin{tabular}{lll}
\hline M de Box & 90,261 \\
\hline F & Aprox. & 14,578 \\
& df1 & 6 \\
& df2 & 72781,380 \\
& Sig. & 0,000 \\
\hline
\end{tabular}

Prueba la hipótesis nula de las matrices de covarianzas de población iguales.

Fuente: elaboración propia (2020)

Seguidamente en la tabla 2 se presentan los autovalores de las funciones canónicas discriminantes, mediante la cual se calculan las desviaciones de las puntuaciones discriminantes entre los grupos respecto a las desviaciones dentro de los grupos. En este caso, la primera función explica el 79,8\% y la segunda el 20,2\%, así mismo en relación a los autovalores se puede afirmar que la función discrimina. Por último, la correlación canónica presentada en la función 1 es mayor a la hallada en la función 2, por lo que la primera función discrimina más que la segunda.

Tabla 2

Autovalores

\begin{tabular}{lllll}
\hline Función & Autovalor & \% de varianza & \% Acumulado & Correlación canónica \\
\hline 1 & $0,207^{\text {a }}$ & 79,8 & 79,8 & 0,414 \\
2 & $0,052^{\text {a }}$ & 20,2 & 100,0 & 0,223 \\
\hline
\end{tabular}

a. Se utilizaron las primeras 2 funciones discriminantes canónicas en el análisis.

Fuente: elaboración propia (2020). 
Con respecto, a la Lambda de Wilks (tabla 3) que representa el poder de discriminación se puede afirmar que este no es muy elevado ya que presenta valores próximos a $1(0,787$ y 0,95). Los grados de libertad de la Chicuadrado son 4 y 1, respectivamente y el nivel de significancia en ambos casos está por debajo de 0,05 por lo que se rechaza la hipótesis nula, es decir, pudiéndose afirmar que al menos 1 de los ejes discriminantes es significativo por lo que se afirma que ambos ejes son significativos.

Tabla 3

Lambda de Wilks

\begin{tabular}{lllll}
\hline & Lambda & de & & \\
Prueba de funciones & Wilks & Chi-cuadrado & gl & Sig. \\
\hline 1 a 2 & 0,787 & 24,756 & 4 & 0,000 \\
2 & 0,950 & 5,273 & 1 & 0,022 \\
\hline
\end{tabular}

Fuente: elaboración propia (2020)

A partir de los datos presentados en la tabla 4 se establecen las funciones discriminantes canónicas siguientes:

1) $D_{1}=0,873 \times n$ o de compañías incorporadas al grupo $+0,594 \times$ ratio fondo de maniobra

2) $D_{2}=-0,5 \times n=$ de compañías incorporadas al grupo $+0,812 \times$ ratio fondo de maniobra

Tabla 4

Coeficientes de función discriminante canónica estandarizada

\begin{tabular}{lcc}
\hline & Función 1 & Función 2 \\
\hline № de compañías incorporadas en el grupo & 0,873 & $-0,500$ \\
Ratio fondo de maniobra & 0,594 & 0,812 \\
\hline \multicolumn{3}{c}{ Fuente: elaboración propia (2020) }
\end{tabular}

Mediante las funciones en centroides de los grupos (tabla 5) se puede observar como las funciones 1 y 2 discriminan según la variable localización, en este caso no hay un punto de corte discriminante ya que el conjunto de las observaciones se encuentra dividido en tres grupos.

Tabla 5

Funciones en centroides de grupo

\begin{tabular}{lcc}
\hline Localización & Función $\mathbf{1}$ & Función 2 \\
\hline Ibiza & 0,468 & $-0,052$ \\
Córdoba & $-0,566$ & $-0,308$ \\
Santiago de Compostela & $-0,297$ & 0,303 \\
\hline
\end{tabular}

Las funciones discriminantes canónicas sin estandarizar se han evaluado en medias de grupos

Fuente: elaboración propia (2020)

A continuación, se calculan las tres funciones de clasificación, mediante las cuales se podrá clasificar a cada empresa en función del grupo cuya función discriminante resulte de mayor valor. En este caso, las funciones de clasificación son:

1) $F_{I B I Z A}=0,129 \times n$ o de compañías incorporadas al grupo $+0,528 \times$ ratio fondo de maniobra - 1,246

2) $F_{\text {CÓRDOBA }}=0,27 \times n$ ㅇ de compañías incorporadas al grupo - 1,368 $x$ ratio fondo de maniobra - 1,706

3) F santiago de compostela $=0,018 \times$ no de compañías incorporadas en el grupo $+0,147 \times$ ratio fondo de maniobra - 1,187 
Tabla 6

Coeficientes de función de clasificación

\begin{tabular}{lccc}
\hline & \multicolumn{3}{c}{ Localización } \\
\cline { 2 - 4 } & Ibiza & Córdoba & $\begin{array}{c}\text { Santiago de } \\
\text { Compostela }\end{array}$ \\
\hline № de compañías & 0,129 & 0,027 & 0,018 \\
incorporadas en el grupo & 0,528 & $-1,368$ & 0,147 \\
Ratio fondo de maniobra & $-1,246$ & $-1,706$ & $-1,187$ \\
(Constante) &
\end{tabular}

Funciones discriminantes lineales de Fisher

Fuente: elaboración propia (2020)

Por último, los resultados del estudio denotan una clasificación donde el 57,9\% de las observaciones están agrupadas correctamente. En relación a los grupos se puede afirmar que la mayoría (76\%) de los hoteles situados en Ibiza están clasificados correctamente. Sin embargo, en el caso de Córdoba solo el 33,3\% se encuentra bien clasificado. Para el caso de Santiago de Compostela, las características de la clasificación denotan que casi la mitad de los casos están bien clasificados (48,5\%), mientras que el $45,5 \%$ se situarían en el grupo de Ibiza. De esta manera, el modelo de clasificación presentado atendiendo a la localización de las Ciudades Patrimonio de la Humanidad ubicadas dentro del grupo de ciudades planeta, se clasifican de acuerdo al fondo de maniobra y al número de compañías, lo que permite clasificar correctamente casi 6 de cada 10 casos.

Tabla 7

Resultados de clasificación

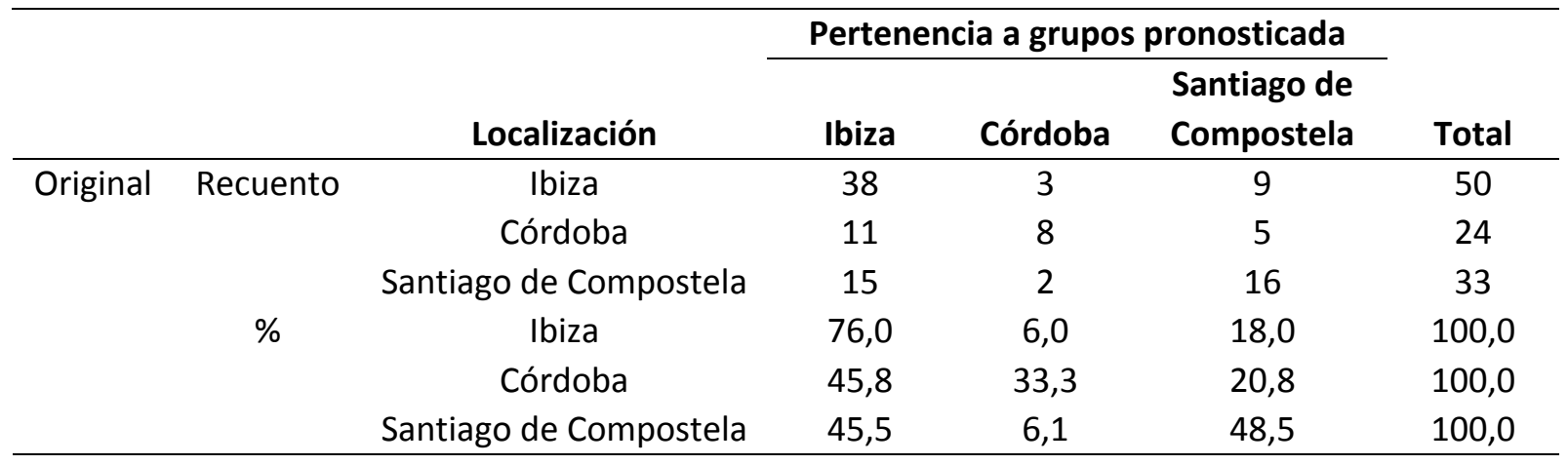

a. $57,9 \%$ de casos agrupados originales clasificados correctamente.

Fuente: elaboración propia (2020)

\section{Conclusiones}

En esta investigación se desarrolla un modelo que permite explicar la clasificación de las empresas hoteleras de las CPHE denominadas como ciudades planeta utilizando la técnica del análisis discriminante multivariado. La variable categórica es la localización existiendo tres grupos: a) empresas hoteleras de Ibiza, b) empresas hoteleras de Córdoba y c) empresas hoteleras de Santiago de Compostela. Respecto a las variables expliativas el modelo utiliza el número de compañías incorporadas al grupo y el fondo de maniobra, de tal manera que a partir de estas variables pueden pronosticar el grupo al cual debería de pertenecer. Este hecho pone de manifiesto que a pesar de que todas las empresas analizadas están situadas en Ciudades Patrimonio de la Humanidad y, concretamente, en ciudades caracterizadas por tener un volumen elevado de turistas, en su mayoría extranjeros, que logran que dichas ciudades presenten un alto indice de ocupación hotelera, que están dotadas de infraestructuras accesibles y cuyo mercado laboral está estrechamente relacionado con el sector turístico. 
Pese a que se trata de ciudades con características similares, existen diferencias entre las tres ciudades tal y como se ha explicado con anterioridad. Concretamente, el caso de Córdoba se trata de un sector turístico el cual se encuentra sustentado en la tipología de turismo cultural fundamentado en el patriminio, al ser la ciudad de España con más monumentos tanto tangibles como intangibles catalogados por la UNESCO como Patrimonio de la Humanidad. Con respecto al sector turístico de Santiago de Compostela, este se caracteriza por basarse en un turismo religioso-cultural al ser el destino final de la Ruta del Camino de Santiago. Mientras que Ibiza fundamenta su turismo en el ocio o vida nocturna.

Es por tanto que, a tres tipologías turísticas se justifica el hecho de que existan tres grupos (Ibiza, Córdoba y Santiago de Compostela) claramente diferenciados afectando a la estructura, diseño, funcionamiento y situación financiera de las empresas. De esta menera, con la clasificación presentada en la presente investigación se pone de manifiesto la existencia de diferencias entre las tres ciudades. En definitiva, se realiza una clasificación de acuerdo a dos variables discriminatorias que permite de una parte explicar dicha clasificación al 57,9\% y además, permite comprobar si una empresa, de acuerdo a sus características, es más afín a un grupo u otro.

De esta manera y pese a que, en un principio, se tomó un número elevado de variables solo las variables fondo de maniobra y número de compañías incorporadas al grupo permiten distinguir sobre la permanencia a un grupo $u$ otro en función de la localización. Este hecho hace que el equilibrio empresarial se convierta en una variable clave respecto a la localización medida a través del fondo de maniobra, es decir, de la diferencia entre el activo circulante y el pasivo corriente. De este modo, se convierte en un elemento diferenciador el hecho de que las empresas dispongan de equilibrio finaniero procedente de sus actividades corrientes una vez que se han cumplido las obligaciones derivadas de su actividad productiva. En el caso objeto de estudio se observa como las empresas localizadas en la ciudad de Córdoba en términos promedios tienen un fondo de maniobra mucho más reducido que el de Ibiza o de Santiago de Compostela, debido a la estructura, funcionamiento y capacidad de las mismas las cuales pueden estar asociadas a la oferta turística siendo en el caso de Córdoba inferior a la de los otros dos destinos y ofertándose de forma específica una sola tipología turística a diferencia del resto de ciudades de estudio donde se conjuga el turismo de masas con el turismo cultural.

Igualmente, ocurre con el número de compañías incorporadas al grupo en donde se manifiesta que para el caso de Santiago de Compostela el número de estas es muy reducido oscila a 1 compañía incorporada al grupo, a diferencia de Ibiza en donde son alrededor de 6 empresas de promedio las que se encuentran incorporas al grupo y de Córdoba en donde alcanza las 2 empresas. Esto puede deberse a la dinámica existente en Santiago de Compostela en donde predominan las empresas individuales de carácter local estrechamente vinculadas al turismo patrimonial y religioso. Por el contrario, en Ibiza predominan los grupos empresariales hoteleros relacionados con el turismo nocturno y de ocio.

\section{Referencias bibliográficas}

Aching, C. (2006). Guía rápida: ratios financieros y matemáticas de la mercadotecnia. El Cid Editor

Azpelicueta, M., Ramón, J. y Abril, M. (2018). La oferta turística de la ciudad de Ibiza en el siglo XXI: Patrimonio de la Humanidad y establecimientos de lujo. HOLOS, 34 (3), 413-428. Recuperado de http://www2.ifrn.edu.br/ojs/index.php/HOLOS/article/view/4341.

Braintrust, (2019). Observatorio turístico del GCPHE 2019. https://www.ciudadespatrimonio.org/publicaciones/ObservatorioTuristicoGCPHE2019.pdf

Cantarero, S., Sanchís, J. y Comeche, J. (2011). El papel del agente de desarrollo local en la creación de empresas en el contexto del desarrollo local. El caso valenciano. Scripta Nova. Revista Electrónica de Geografía y Ciencias Sociales, 15 (358). Recuperado de http://www.ub.es/geocrit/sn/sn-358.html 
Cárdenas-García, P.J.; Pulido-Fernández, J.I. y Mudarra-Fernández, A.B. (2015). Direct economic impact of tourism on World Heritage Cities: An approach to measurement in emerging destinations. Czech Journal of Tourism, 3(2), 91-106. Recuperado de https://content.sciendo.com/view/journals/cjot/3/2/articlep91.xml

Carvalho, K. y Moquete, S. (2011). El turismo en la dinámica territorial. Estudios y Perspectivas en Turismo, 20, $441-461$. Recuperado de https://dialnet.unirioja.es/servlet/articulo?codigo=3738537

Choi, M. y Lee, C. (2020). The Peter Pan síndrome for small and médium-sized enterprises: Evidence from Korean manufacturing firms. Management Decision Economics, 41, 426-4445. Recuperado de https://onlinelibrary.wiley.com/doi/full/10.1002/mde.3111?casa_token=ILd_u9RMaLMAAAAA\%3Ak2aqEhGF00S4BW17mHr8UGKZRHHBIP81t9ImnyEWXFis3bJm02CcrOIDDnCxEZU7OF4um6BfFfWDg

Corona, R., Bejarano, V. y González, G. (2017). Análisis de estados financieros individuales y consolidados. UNED - Universidad Nacional de Educación a Distancia

García-Gallego, A. y Mures-Quintana, M. (2013). La muestra de empresas en los modelos de predicción de fracasos: influencia en los resultados de clasificación. Revista de métodos cuantitativos para la economía y la empresa, (15), 133-150. Recuperado de https://www.upo.es/revistas/index.php/RevMetCuant/article/view/2226

García-Londoño, M.L.; González-Fresneda, S.L. y Acevedo-Velandia, F. J. (2019). Rendimientos y riesgos de las estrategias de sostenibilidad para el desarrollo turístico del Valle de Cocora (Colombia). Revista Espacios. 40(6), página 17. Recuperado de: https://revistaespacios.com/a19v40n06/a19v40n06p17.pdf

Gil, A. (2004). Nuevas estrategias para el análisis financiero de la empresa. Editorial Ariel

Gómez-Bezares, F., Madariaga, J. y Santibáñez, J. (2004). Modelos integrados de análisis financiero mediante ratios. Ediciones Deusto - Planeta de Agostini Profesional y Formación S.L.

González, F. y López-Guzmán, T. (2016). El desarrollo turístico en la ciudad de Córdoba a partir del Patrimonio Cultural Inmaterial. Ge-conservación, 9, 96-106. Recuperado de https://dialnet.unirioja.es/servlet/articulo?codigo=5590163

Hernández, R., Fernández, C. y Baptista, P. (2014). Metodología de la investigación. (6a ed.) México: Mc Graw Hill.

Hontalvilla, A.; Lado, R. y Vivel, M. (2018). Sector hotelero español y riesgos financieros: un análisis de Meliá Hotels International S.A. Revista Espacios. 39(6). Recuperado de http://www.revistaespacios.com/a18v39n06/a18v39n06p26.pdf.

Hurtado, J. (2010). Metodología de la investigación: guía para la comprensión holística de la ciencia. (4ạ. Ed). Caracas, Venezuela: Ediciones Quirón.

Jenny, F., \& Weber, A. (1976). Profit rates and structural variables in French manufacturing industries. European Economic Review, 7, 187- 206. Recuperado de https://www.sciencedirect.com/science/article/abs/pii/0014292176900581

Lado, R.; Otero, L. y Vivel, M.(2014). Impacto de la localizacion y la estructura de mercado en la rentabilidad de los establecimientos hoteleros. Tourism \& Management Studies, 10 (2), 41-49. Recuperado de http://www.scielo.mec.pt/scielo.php?pid=S2182-84582014000200006\&script=sci_arttext\&tlng=es. 
López, A. y De Esteban, J. (2010). El turismo sostenible como dinamizador local. Observatorio Medioambiental, 13, 109-129. Recuperado de https://revistas.ucm.es/index.php/OBMD/article/view/OBMD1010110109A

López, R. (2006). Análisis financiero para la determinación de quiebra. Encuentro, 38 (74), 80-96. Recuperado de https://lamjol.info/index.php/ENCUENTRO/article/view/3713

Martínez-Caraballo, H.R.; Cazallo-Antúnez, A.M.; Meñaca-Guerrero, I. y Uribe-Urán, C.M. (2020). Desempeño financiero de las empresas minoristas de alimentos y bebidas en Barranquilla - Colombia. Revista de Ciencias Sociales, 26, (1), 144-160. Recuperado de https://produccioncientificaluz.org/index.php/rcs/article/view/31316/32366

Martínez, O. y García, R. (2016). La contribución de las ciudades patrimonio de la humanidad al desarrollo turístico: análisis de caso de Santiago de Compostela. International Journal of Scientific Management and Tourism, 2, (1), 119-128. https://dialnet.unirioja.es/descarga/articulo/5665911.pdf

Moral-Cuadra, S.; López-Felipe, M. T. y Menor-Campos, A. y Rueda-López, R. (2020). Dimensiones motivacionales y perfil sociodemográfico en una ciudad Patrimonio de la Humanidad: el caso de la ciudad de Córdoba (España). Revista Espacios. 41(29), página 8. Recuperado de: http://ww.revistaespacios.com/a20v41n29/a20v41n29p08.pdf

Morales-Fernández, E. y Lanquar, R. (2014). El futuro turístico de una ciudad patrimonio de la humanidad: Córdoba 2031. Tourism \& Management Studies, 10 (2), 7-16. Recuperado de http://www.scielo.mec.pt/scielo.php?script=sci_arttext\&pid=S2182-84582014000200002

Narváez, E. (2014). El turismo alternativo: una opción para el desarrollo local. RevllSE: Revista de Ciencias Sociales y Humanas, 6 (6), 9-18. Recuperado de https://dialnet.unirioja.es/servlet/articulo?codigo=5537859

Neumann, M., Böbel, I., \& Haid, A. (1982). Innovations and market structure in West German industries. Managerial and Decision Economics, 3, 131- 139. Recuperado de https://onlinelibrary.wiley.com/doi/abs/10.1002/mde.4090030305

Nevado, D. y López, V. (2009). Proyecciones de la econometría sobre la empresa: un modelo de previsión sobre indicadores financieros. D - Fundación General de la Universidad Autónoma de Madrid.

Ortega, J. (2010). Análisis de estados financieros: teoría y aplicaciones. Editorial Desclée de Brouwer

Otzen, T. y Manterola, C. (2017). Técnicas de muestreo sobre una población a estudio. Int. J. Morphol, 35 (1), 227-232. Recuperado de https://scielo.conicyt.cl/pdf/ijmorphol/v35n1/art37.pdf

Pérez, J., Medina-Viruel, M., López-Guzmán, T. y Muñoz-Fernández, G. (2020). Segmentación y percepción turística en destinos patrimonio material de la humanidad: Córdoba (España). Revista de Ciencias Sociales, 26(1), 11-24. Recuperado de https://dialnet.unirioja.es/servlet/articulo?codigo=7384402

Porter, M. (2008). The five competitive forces that shape strategy. Harvard Business Review, 1 (1), 23-40.

Puerta, F., Vergara J. y Huertas, N. (2018). Análisis financiero: enfoques en su evolución. Criterio Libre, 16 (28), 85-104. Recuperado de https://dialnet.unirioja.es/servlet/articulo?codigo=6676021

Raguseo, E.; Vitari, C. y Pigni, F. (2020). Profiting from big data analytics: The moderating roles of industry concentration and firm size. International Journal of Production Economics, 229, 1-12. Recuperado de https://www.sciencedirect.com/science/article/pii/S0925527320301420?casa_token=sXq- 
9YF1TgkAAAAA:9Sfm5saSNVnyh9He3B5ArdbGgeQrP3y1uLTQMehjkBHczU1tLYT1HUI4b1RQ6PrmRBZ4Lrhn JW

Rico, P. (2015). Análisis económico-financiero de las empresas concesionarias de autobuses en España. Revista de métodos cuantitativos para la economía y la empresa, 20, 95-111. Recuperado de https://www.econstor.eu/handle/10419/174231

Ricoy-Lorenzo, C. (2006). Contribución sobre los paradigmas de investigación. Educação, 31(1), 11-22.

Recuperado de https://www.redalyc.org/articulo.oa?id=1171/117117257002

SABI (2020). Base de datos SABI: Sistema de Análisis de Balaces Ibéricos.

Secall, R. (2010). La competitividad de las zonas turísticas. Universidad de Málaga. Recuperado de https://eco.mdp.edu.ar/cendocu/repositorio/00019.pdf.

Wallingre, N. (2007). Limitaciones o beneficios del desarrollo local en el turismo. Tiempo de gestión, 3, 11-20. Recuperado de https://repotur.yvera.tur.ar/handle/123456789/4311

Esta obra está bajo una Licencia Creative Commons
Attribución-NoCommercial 4.0 International
\[ \text { (cc) BY-NC } \]

